\title{
Carga laboral y nivel de estrés del personal de enfermería en León, Nicaragua
}

\author{
Workload and Stress Level of Nursing Staff in León, Nicaragua
}

\author{
Lester Fidel García Guzmán ${ }^{1}$, Katherin Yanina Carrión Silva ${ }^{2}$
}

${ }^{1}$ Escuela de Enfermería, Facultad de Ciencias Medicas UNAN León, Nicaragua

${ }^{2}$ Estudiante de Licenciatura en Enfermería Mención materno infantil. Escuela de Enfermería, Facultad de Ciencias Medicas UNAN León, Nicaragua Contacto: lester.garcia@cm.unanleon.edu.ni

Fecha de recepción: 25 de mayo de 2018 / Fecha de aceptación: 09 de noviembre de 2018

\begin{abstract}
Resumen
Enfermería es una de las labores más humanitarias, pero también es considerada una de las de mayor riesgo de padecer estrés por la asignación que esta profesión demanda; por esto se consideró importante iniciar una investigación teniendo como objetivo determinar la carga laboral en relación al nivel de estrés del personal de enfermería del centro de salud Félix Pedro Picado, León, III trimestre 2016.

El estudio fue descriptivo de corte transversal, se utilizó la recolección y el análisis a través de preguntas con respuestas cerradas; se desarrolló en el centro de salud cabecera Félix Pedro Picado, ubicado en el municipio de León, barrio Sutiaba, analizando al personal de enfermería activos del centro, totalizando 26 personas en estudio, pretendiendo determinar la relación entre la carga laboral y nivel de estrés. Como instrumento se implementó la escala de estrés percibido (pss14); el análisis fue mediante el paquete estadístico SPSS v20 aplicando estadística descriptiva.

En los hallazgos se obtuvo que la mayoría de la población está en edades de 20-30 años, son técnicos superior de enfermería, con 1-10 años de labor, presenta sobre carga laboral que desencadena estrés moderado, el cual afecta a la población en sus labores encontrando factores relacionados entre sí como la edad, horas extras, falta de organización, falta de compañerismo.
\end{abstract}

Palabras clave: carga laboral, enfermería, estrés.

\begin{abstract}
Nursing is one of the most humanitarian tasks but it is also considered one of the most risky to suffer stress due to the allocation that this profession demands, for this reason it was considered important to start an investigation with the objective of determining the workload in relation to the level of stress of the nursing staff of the health center Felix Pedro Picado, León, III quarter 2016.

The study was descriptive of cross section, the collection and analysis was used through questions with closed answers, it was developed in the health center Félix Pedro Picado, located in the municipality of León, suburb Sutiaba, analyzing the nursing staff assets of the center, totaling 26 people under study, trying to determine the relationship between workload and stress level. As an instrument the scale of perceived stress was implemented (pss14); the analysis was through the statistical package SPSS v20 applying descriptive statistics.

The findings showed that the majority of the population is between 20 and 30 years old, they are senior nursing technicians, with 1-10 years of work, they present workload that triggers moderate stress, which affects the population in their work finding related factors such as age, overtime, lack of organization, lack of companionship.
\end{abstract}

Keywords: workload, nursing, stress.

\section{Introducción}

La enfermería es un servicio público que contribuye a preservar la vida y la salud de las personas desde la perspectiva humana, ética, interpersonal y terapéutica. La característica singular de la práctica de enfermería es el cuidado de las personas (individuo, familia, comunidad, grupo y sociedad) que interactúan con su entorno, por lo que se ve comprometida en todos los ámbitos con la población, generando así una carga laboral para el personal. 
La carga laboral es el conjunto de requerimientos a los que se ve sometida la persona a lo largo de su jornada laboral desencadenando niveles de estrés. El estrés es una de las manifestaciones más características de la vida moderna y una de las causas principales de agotamiento del organismo.

Las condiciones laborales actuales involucran modelos de producción y prestación de servicios con características de trabajo aceleradas e intensificadas. Los modelos determinan el aumento de la productividad, mediante la combinación del ritmo de trabajo, de la carga de responsabilidad y de la reducción de los intervalos de descanso en la jornada de trabajo.

En este sentido, la jornada de trabajo constituye una dimensión importante en la calidad de empleo, influyendo en la seguridad y salud del trabajador, en los aspectos personales y familiares, y también en la organización laboral dentro de la institución.

Enfermeros estresados están más susceptibles a la ocurrencia de accidentes y enfermedades relacionadas al trabajo y pueden además desarrollar sus actividades de forma ineficiente, resultando ciertamente en consecuencias negativas al individuo y/o a la población asistida.

Se añade a esta problemática la cuestión de la alta carga horaria que los trabajadores del área de la salud, inclusivo los de enfermería, suelen llevar a cabo, haciéndoles trabajar de forma excesiva. El exceso de trabajo parece favorecer enfermedades mentales y/o físicas en trabajadores del área de la salud, además de facilitar la ocurrencia de absentismos, accidentes de trabajo, agotamiento, sobrecarga laboral y ausencia de ocio.

Enfermeros, a fin de superar las adversidades de su trabajo, buscan motivación, como el dinero y el conocimiento, para seguir una doble jornada laboral, desafiando los factores extrínsecos e intrínsecos que surgen constantemente.

En el área de la salud, el desempeño de los profesionales es acompañado por la expectativa de altos niveles de competencia y de responsabilidad en su trabajo pero la sobrecarga de responsabilidades con llevan a no realizar un trabajo satisfactorio frustrando así a todo el personal de la salud y afectando a la población en general a la que le sirven.

\section{Objetivo}

Determinar la carga laboral en relación al nivel de estrés del personal de enfermería del centro de salud Félix Pedro Picado, en León, durante el III trimestre, 2016.

\section{Objetivos especificos}

- Caracterizar demográficamente la población en estudio.

- Describir la carga laboral del personal de enfermería.

- Identificar el nivel de estrés del personal de enfermería.

\section{Materiales y Métodos}

Tipo de estudio: El estudio es descriptivo de corte transversal.

Área de estudio: El estudio fue el centro de salud cabecera Félix Pedro Picado, ubicado en el municipio de León, barrio Sutiaba. Este centro de salud cuenta con servicios de atención primaria, que abarca principalmente promoción de la salud y prevención de las enfermedades, la que se divide en educación en salud $\mathrm{y}$ atención en los programas prioritarios, que son: control prenatal, planificación familiar, detección de cáncer cervicouterino, atención al parto bajo riesgo, puerperio, inmunización, atención integral al niño, vigilancia crecimiento y desarrollo, micronutrientes, inmunización y terapia de rehidratación oral. Cuenta con enfermeras asistenciales que laboran las 24 horas.

Periodo de estudio: III Trimestre 2016

Unidad de análisis: Personal de enfermería activos en la unidad de salud, que laboran en los diferentes puestos que conforma el personal del centro de salud de Sutiaba y brindan atención las 24 horas al día, siendo los turnos de trabajo de las enfermeras rotativos en los siguientes horarios: 7:30 a.m. $-2: 00$ p. m., 2:00 p. m. $-9: 00$ p. m., y 9:00 p. m. $-7: 00$ a.m.

Población de estudio: Esta investigación fue de 35 enfermeras que laboran en la unidad de salud en estudio. 


\section{Criterio de inclusión}

- Personal de enfermería activa del centro de salud Félix Pedro Picado.

- Edades: 20-50 años.

- Ambos sexos.

- Que este actualmente como personal activo en la unidad de salud.

- Personal de diferentes áreas de atención.

Fuente de información: Primaria, entrevistas; secundaria, control de asistencia del personal, rol de turno dirección de la unidad de salud y jefatura de enfermería.

Variables de estudio: nivel de estrés, carga laboral.

Instrumento de recolección de datos: Escala de estrés percibido (pss14), la cual ha sido validad internacionalmente y su última aplicación fue por el centro de investigaciones en demografía y salud CIDS en el año 2015. Esta escala está diseñada para medir el grado de estrés psicológico en la población; la sumatoria produce la puntuación total de la escala, nos brinda valores con un rango entre 0 y 56 puntos: cuanto más alta la puntuación, mayor grado de estrés.

El instrumento sobre datos sociodemográficos y carga laboral fue diseñado por los autores, está compuesto por preguntas cerradas.

Procedimiento de recolección de datos: Solicitud de autorización institucional para la realización de entrevistas al personal de enfermería, procediendo a visitar la unidad de salud para desarrollar la entrevista en horas laborales, para contar con la participación de todo el personal activo del puesto de salud.

Se realizó entrega y firma del consentimiento informado a los participantes del estudio; a los individuos que aceptaron formar parte de la investigación se les entrego el formulario para ser llenado por ellos mismos.

Procesamiento y Análisis de la información: La información se analizo a través del paquete estadístico SPSS v20, aplicando estadística descriptiva, potenciando el uso de las frecuencias absolutas y relativas y presentando los resultados a través de gráficas y tablas para mejorar la comprensión por parte del auditorio.

Aspectos éticos: Consentimiento informado, anonimato, confidencialidad y autonomía.

\section{Resultados}

En este estudio se contó con un total de 26 individuos, que representan al $100 \%$ de la población, encontrando los siguientes resultados: con respecto a la edad, el $35 \%$ de los participantes se encuentra entre las edades de 20-30 años, seguido de un 31\% en edades de 31-40, un $15 \%$ con las edades de $41-50$, y un $19 \%$ con las edades de 51-60 años; en estado civil, el 42\% son casadas; según su procedencia, 5 (19\%) son del área rural, $21(81 \%)$ son del área urbana. Según el número de hijos, el $46 \%$ tienen entre 1-3 hijos; según el título, el $27 \%$ son auxiliares de enfermería, un $38 \%$ son técnicos superiores de enfermería, y un $35 \%$ son licenciadas en enfermería.

Con respecto a los años de labor del personal de enfermería en el centro de salud, encontramos los siguientes datos: el $38 \%$ tienen de 2-10 años de labor, un 27\% 11-20 años de labor, y el 27\% 21-30 años de labor. El $35 \%$ de las enfermeras trabajan más de 40 horas semanales; un $73 \%$, entran a sus labores a las 7 a. m., y un $69 \%$ culminan su jornada laboral a las 5 p.m. Se encontró que el $77 \%$ de las enfermeras realizan horas extras; al consultar si son reconocidas las horas extras en términos salariales, el $88 \%$ respondieron que no.

El38\% de la población de estudio frecuentemente se siente cansada o fatigada; el $50 \%$ consideran el clima organizacional como inadecuado. Al consultar sobre el reconocimientos de los logros laborales obtenidos, un $69 \%$ respondieron que no son reconocidos; el $42 \%$ de las enfermeras refirió que se presenta favoritismo en las asignaciones de labores del personal.

En el cruce de variables se encontró que el $36.6 \%$ de las enfermeras que realizan horas extras presentan estrés moderado y un $26.9 \%$ presentan estrés severo.

Respecto al clima organizacional del personal de enfermería, se obtuvo que, de los que lo consideran "Inadecuado", un $23.1 \%$ presentan estrés moderado y un $15.4 \%$ presentan estrés severo.

\section{Discusiones}

Al realizar la contingencia entre edad y nivel de estrés, se obtuvo que el nivel de estrés severo aumenta su prevalencia en la población de mayores edades (5160 ), lo que se reafirma con lo propuesto por María José Vidal García en su curso de seguridad y salud en el 


\begin{tabular}{|l|l|c|c|}
\hline Variable & Indicador & $\mathbf{N}^{\circ}$ & $\%$ \\
\hline Atención directa al usuario & Nunca & 1 & 4 \\
& Muy poco frecuente & 11 & 42 \\
& Frecuente & 14 & 54 \\
\hline Atención y administración de farmacia & Nunca & 8 & 31 \\
& Muy poco frecuente & 8 & 31 \\
& Frecuente & 10 & 38 \\
\hline Visita domiciliar a pacientes & Nunca & 3 & 12 \\
& Muy poco frecuente & 8 & 31 \\
& Frecuente & 15 & 58 \\
\hline Educación continua & Nunca & 6 & 23 \\
& Muy poco frecuente & 12 & 46 \\
& Frecuente & 8 & 31 \\
\hline Trabajos administrativos & Nunca & 1 & 4 \\
& Muy poco frecuente & 7 & 27 \\
& Frecuente & 18 & 69 \\
& Total & 26 & 100 \\
\hline
\end{tabular}

Tabla 1. Frecuencia de las actividades realizadas por el personal de enfermería del puesto de salud Félix Pedro Picado

\begin{tabular}{|l|c|c|}
\hline Nivel de estrés & Frecuencia & Porcentaje válido \\
\hline Sin estrés & 6 & 23 \\
\hline Estrés moderado & 10 & 38 \\
\hline Estrés severo & 10 & 38 \\
\hline Total & 26 & 100 \\
\hline
\end{tabular}

Tabla 2. Nivel de estrés del personal de enfermería del puesto de salud Félix Pedro Picado

trabajo, que la población que labore en edades que sobrepasen los 60 años presentarán mayor dificultad al grado de movilización, el esfuerzo que debe realizar para llevar acabo la tarea determinada, la concentración..., y esto desencadenará una frustración, fatiga y aumentará el nivel de estrés.

En cuanto las funciones laborales de acuerdo a su formación académica, se encontró que los que poseen título auxiliar o técnico superior consideran que sus funciones laborales no corresponden a su formación académica. Este fenómeno es común, de acuerdo a lo observado en otros estudios, donde para estimular a los buenos profesionales el empleador les aumenta su responsabilidad laboral, no son capaces de afrontarla y se estresan.
En los años de labor en el puesto de salud, predominó desde 2 a 10 años laborales, por lo cual consideramos que es una población laboralmente activa en todas sus capacidades profesionales, retomando esto en base a lo propuesto por Vidal García, para quien La población joven en edades aptas para laborar no presentarán complicación alguna, pues presentan todas las condiciones físicas que se requieren.

En cuanto a las horas laborales por semana, se obtuvo que el $35 \%$ de la población trabaja más de 40 horas semanales, el $16 \%$ de la población realiza 1-2 turnos por semana; respecto a la hora de entrada, varía de 7 a.m., 11 a.m. y 9 p.m., y la hora de salida mayormente predominó de 3-5 p. m. 
Esto concuerda con datos expuesto por la Organización Panamericana de la Salud en cuanto a la profesión, que la jornada laboral de enfermería más difundida es de 8 horas diarias y 45 semanales; además, las condiciones de trabajo de las enfermeras se caracterizan por la sobrecarga de trabajo, jornadas extensas, turnos rotatorios, trabajo nocturno, frecuentes cambios de servicios y carga psicológica por el manejo de situaciones críticas.

De acuerdo a los resultados del tiempo para almorzar y la hora designada, se obtuvo que el $65 \%$ de la población tiene tiempo para almorzar y un $77 \%$ tiene 30 minutos designado para su almuerzo, lo que beneficia a los trabajadores, según lo que plantea la Organización Internacional del Trabajo, que la alimentación es fundamental para todo trabajador; esto fortalece su cuerpo y es provechoso, aparte beneficia a los trabajadores siendo más productivos. Al realizar la contingencia con el nivel de estrés, se obtuvo que la población que le asigna media hora para almorzar, el $30.8 \%$ manifiestan estrés moderado y un $30.8 \%$ estrés severo.

Se evidenció que un $62 \%$ de la población dispuso que las relaciones entre sus compañeros de labores son competitivas, por lo que Vidal García plantea que como consecuencia de esta mala relación entre el grupo de trabajo va a verse perjudicado el rendimiento laboral.

En cuanto a la existencia de un programa para tratar el estrés, se obtuvo que el $81 \%$ de la población manifestó que no existe ningún programa, lo que manifiesta Carolina Pinna, que toda empresa debe tener un área para tratar a su personal, no pasar por alto cada uno de los problemas, ya que esto disminuye la productividad, aumenta el absentismo, aumenta las quejas de clientes, etc.

Al analizar el nivel de estrés de la población en estudio, se obtuvo que la mayoría de la población se encuentran con: sin estrés $23.1 \%$, estrés moderado $38.5 \%$ y estrés severo $38.5 \%$. Según Ignacio de la Fuente plantea que estos dos últimos niveles de estrés representan una emoción conflictiva en un entrampamiento físico o psíquico (estrés incorrecto o malo), conocido como distrés.

De igual manera, las que realizan horas extras presentan un $34.6 \%$ de estrés moderado. Vidal García plantea que realizar horas extras por una sobrecarga laboral, trabajos pendientes a entregar de forma inme- diata, mala organización del tiempo..., se pierde el control y el ánimo se frustra, manifestando así estrés laboral.

Al realizar la contingencia en si presentan cansancio o fatiga en sus labores con el nivel de estrés, se encontró que el $23.1 \%$ de la población que frecuentemente se fatiga o cansa presenta estrés moderado, y el $23.1 \%$ de la población que muy poco frecuentemente se fatiga presenta estrés severo. El Dr. Camín expresa que el estado de fatiga conlleva a un sentimiento de fracaso, con falta de interés hacia las personas que tenemos a nuestro cuidado o falta de interés hacia la tarea que tenemos asignada.

\section{Conclusiones}

El personal de enfermería del puesto de salud Félix Pedro Picado se caracteriza por ser una población en donde predomina el sexo femenino; el grupo etario predomina la población de 20-30 años, la mayor parte de las mujeres son solteras, provienen del área urbana, la mayoría no tienen hijos y predominan más las técnico superior de enfermería.

Dentro de la carga laboral, se encontró como dato relevante que la mayoría de la población en estudio inician sus labores a las 7:00 a.m., no tiene horas de salida fija, la mayoría manifiesta salir más tarde de su hora normal, realiza horas extras sin ser reconocidas, la mayor parte son multifuncionales, realizan sus labores rotatoriamente, manifiestan fatiga o cansancio poco frecuente en sus jornadas laborales.

El clima organizativo es inadecuado, se presenta favoritismo a la hora de asignación de labores y no son reconocidos sus logros obtenidos; la mayoría consideran que la relación con sus compañeros es competitiva, por lo que no hay un trabajo en equipo formidable que trabajen en conjunto por un mismo objetivo; también se encontró que no se cuenta con algún programa de prevención y tratamiento del estrés.

Al identificar el nivel de estrés mediante la escala de estrés percibido (pss14), se obtuvo que una parte significativa de la población se encuentra con niveles de estrés severo y moderado, en cambio una minoría de la población se encuentra sin estrés.

Las variables de carga laboral que presentaron relaciones con el estrés severo fueron las siguientes: la edad, funciones laborales en correspondencia a su 
formación académica, horas extras, tiempo designado para almorzar corto, sentimiento de fatiga y cansancio, clima organizacional y las relaciones laborales.

\section{Recomendaciones}

A partir de los resultados obtenidos en esta investigación, se considera necesario las siguientes recomendaciones.

\section{A jefatura de enfermería:}

- Incluir indicadores del estrés y carga laboral en la vigilancia de la salud de los trabajadores a través de una evaluación periódica de los mismos para prevenir esta problemática.

- Adecuar los cronogramas de trabajo para distribuir la carga equitativa en el personal y dejar espacios necesarios para concluir tareas pendientes o responder a asignaciones nuevas de forma imprevista.

- Adecuar horarios que repercutan lo mínimo en las labores externas al trabajo. Los horarios rotatorios es conveniente que se puedan predecir con tiempo suficiente y que sean estables.

- Implementar programas dirigidos al personal de enfermería con la finalidad de disminuir los factores de carga laboral y minimizar así los factores de riesgo del estrés.

\section{Para el personal de enfermería:}

- Practicar actividades de recreación en su tiempo fuera de la unidad de salud, puesto que estar ocupado en algo que nos entretiene nos hace olvidar los problemas. El ocio nos ayuda a eliminar el estrés acumulado. Buscar nuevos hobbies que permitan desconectar con el trabajo.

- Afianzar la confianza y el trabajo en equipo, trabajar todos juntos con el mismo objetivo de brindar una atención integral.

- Establecer un sistema de triaje en las labores y realizarlas iniciando por las de mayor prioridad y finalizar con las menos prioritarias. Saber qué trabajo tiene prioridad. Si no lo sabemos, nos arriesgamos a realizar varios a la vez y que salgan mal.

\section{A futuros investigadores:}

- A retomar la investigación de manera productiva para enriquecer esta problemática que actualmente afecta al personal de enfermería. Realizar investigaciones con mayor nivel de análisis en esta proble- mática y establecer los factores de riesgo del desarrollo de estrés en esta población para implementar programas preventivos adecuados.

\section{Bibliografía}

1. T. Xavier Estresse: causas e consequências. Rev Perfil Online. [Internet]; 2010. [acesso 20 jul 2010].

2. França Ferrari R. Burnout Syndrome and the socio-demographic aspects of nursing professionals. Acta Paul Enferm. 2012.

3. Lic. Hernández Mendoza Helena. "Estrategias de Afrontamiento ante el estrés laboral en Enfermería" .Revista de enfermería; 15(3):161-166. México 2007.

4. Nursing Workload and Patient Care consejo internacional de enfermaros Última actualización el Lunes 05 de Noviembre de 2012 15:05

5. Grupo del banco mundial 2016, Atlas mundial de la fuerza laboral sanitaria.

6. La Federación Canadiense de Enfermeras 2010-2012 CFNU.

7. Lange I. et al (2000). La práctica de enfermería en América Latina. Documento preparado para la reunión Internacional de la Fundación W. K. Kellogg y OPS "Impacto de Enfermería en Salud de América Latina y el Caribe". Universidad Federal de Mina Gerais; Bello Horizonte.

8. Wright M. G. Garzón N (2006). Análisis crítico holístico de los programas de posgrado en enfermería en América Latina. En: Enfermería en las Américas. OPS Publicación científica 571; Washington.

9. Malagris LEN, Fiorito ACC. Avaliação do nível de stress de técnicos da área de saúde. Estud. psicol. (Campinas), dez. 2006; 23(4): 391-39

10. Aguirre-gas H. (1990) Evaluación de la calidad de la atención médica: Expectativas de los pacientes y de los trabajadores de las unidades médicas. En: Salud Pública de México; 32 (2):170180. México.2013

11. Morales J. M. (2004) Investigación de resultados en enfermería: el camino hacia la efectividad de los cuidados, España.

12. Oficina Regional para las Américas de la Organización Mundial de la Salud

13. La enfermería más allá de los años 2000 informe de un grupo de estudio de la OMS ginebra organización mundial de la salud 2007 series de informes técnicos n842

14. Deyall, T. (2003) Hasta la vista paraíso. En: Revista Perspectivas de Salud, de la Organización Panamericana de la Salud. 8(2), Washington.

15. Organización Panamericana de la Salud (2004). Áreas críticas y prioridades en el desarrollo de enfermería en las Américas. Informe de avance de investigación. Washington. (Sin publicar).

16. Síntesis de las características de las reformas y sus efectos en el personal Dussault y Rigoli (2002)

17. DCOMM ALIMENTACION EN EL TRABAJO ORGANIZACIÓN INTERNACIONAL DEL TRABAJO (O.I.T) 1 DE DICIEMBRE 2005

18. Dr.Enrique Camin Amadon MAZ departamento de prevención "el estrés y riesgo para la salud" año 2012.

19. maría José Vidal García curso de seguridad y salud en el trabajo en la universidad de valencia publicado 5 de marzo 2015.

20. Organización Panamericana de la Salud (2004). Informe del III Simposio Internacional de Políticas y Regulación de Recursos Humanos de Enfermería. Panamá. (Sin publicar)

21. 20-Organización Panamericana de la Salud (2002). Informe de la I Jornada Internacional de de Investigación en Recursos Humanos de Enfermería. México. (Sin publicar)

22. Organización Panamericana de la Salud (2002). Servicios de enfermería para contribuir al logro de la equidad y la calidad en los servicios de salud. Plan de mediano plazo 2002-2005. Washington. 
23. Guevara, E.B. y Mendias, E. (2001) "La reforma del sector salud y sus implicaciones para la práctica, la regulación y la educación de enfermería: un estudio de cinco países". Programa de Recursos Humanos (HSR), Serie 27 OPS/OMS, Washington.

24. Casas J, Repullo JR, Lorenzo S. “Estrés laboral en el medio sanitario y estrategias adaptativas de afrontamiento". Rev Calidad Asistencial 2002.

25. "Sociedad española para el estudio de la ansiedad y el estrés; S.E.A.S" (sede Web). Facultad de Psicología. Universidad Complutense de Madrid. (Acceso en Septiembre 2011).

26. GIL MONTE R. y Peiró J.M. Desgaste Psíquico en el Trabajo. El Síndrome de quemarse. $2^{\circ}$ Edición España.

27. Arriagada, J et al (2000). Recurso de Enfermería: Contribución al proceso de desarrollo. W.KKellogg/Real; Bogotá.

28. Ríos-Risque M.l.; Godoy-Fernández C "Relación entre satisfacción laboral y salud general percibida en profesionales de enfermería de urgencias". Enferm. Clin. 2008; 18 (3):134-41.

29. SELIGMAN ,Labrador F. El Estrés y Nuevas Técnicas de Control. España: Ed. Ternas de Control.2008\}

30. Javier Miravalles Gabinete Psicológico - San Juan de la Cruz 11, 2 Izq, Zaragoza www.javiermiravalles.es

31. Escuela de las necesidades (Virginia Henderson); Escuela de la interacción (Hildegard Peplau); Escuela de los cuidados humanizados (Jean Watson); Escuela de los efectos deseables (Calixto Roy), Enfoques de las relaciones salud - sociedad para el trabajo en salud pública y enfermería comunitaria.

32. MARCELO GARCIA, JUAN ARAGON Y MARCELA ROCERO art.14 título enfermería nosocomial en el hospital miliar ecuador. Pg.34 año 2009.

33. carolina pinna A., Giovanna Riffo carga laboral en enfermería publicada octubre 2013 